Research in Astron. Astrophys. 2010 Vol. X No. XX, 000-000

http://www.raa-journal.org http://www.iop.org/journals/raa

$\boldsymbol{R}$ esearch in

Astronomy and

Astrophysics

\title{
Why Are Halo Coronal Mass Ejections Faster?
}

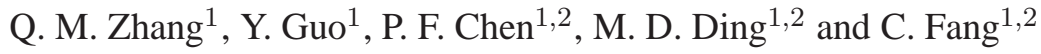 \\ ${ }^{1}$ Department of Astronomy, Nanjing University, Nanjing 210093, China; chenpf@nju.edu.cn \\ ${ }^{2}$ Key Lab of Modern Astron. and Astrophys., Ministry of Education, China
}

Received [year] [month] [day]; accepted [year] [month] [day]

\begin{abstract}
Halo coronal mass ejections (CMEs) were found to be significantly faster than normal CMEs, which was a long-standing puzzle. In order to solve the puzzle, we first investigate the observed properties of 31 limb CMEs that display clearly loop-shaped frontal loops. The observational results show a strong tendency that slower CMEs are weaker in the white-light intensity. Then, we perform a Monte Carlo simulation of 20000 artificial limb CMEs that have average velocity of $\sim 523 \mathrm{~km} \mathrm{~s}^{-1}$. The Thomson scattering of these events is calculated when they are assumed to be observed as limb and halo events, respectively. It is found that the white-light intensity of many slow CMEs becomes remarkably reduced as they turn from being viewed as a limb event to as a halo event. When the intensity is below the background solar wind fluctuation, it is assumed that they would be missed by coronagraphs. The average velocity of "detectable" halo CMEs is $\sim 922 \mathrm{~km} \mathrm{~s}^{-1}$, very close to the observed value. It also indicates that wider events are more likely to be recorded. The results soundly suggest that the higher average velocity of halo CMEs is due to that a majority of slow events and a part of narrow fast events carrying less material are so faint that they are blended with the solar wind fluctuations, and therefore are not observed.
\end{abstract}

Key words: Sun: coronal mass ejections (CMEs) — Sun: activity — methods: numerical - solar-terrestrial relations

\section{INTRODUCTION}

Since the first recognition of coronal mass ejections (CMEs, initially called coronal transients) by OSO-7 (Tousey, 1973), more than 10000 such energetic events have been identified by groundbased and space-borne coronagraphs. Although remarkable progress had been made before, the Large Angle Spectrometric Coronagraph (LASCO) (Brueckner et al., 1995), which is aboard the Solar and Heliospheric Observatory (SOHO) spacecraft (Domingo et al., 1995) launched at the end of 1995, revolutionized our understanding of this eruptive activity for its large field of view (FOV), increased sensitivity and dynamic range. CMEs are often associated with solar flares and filament eruptions (Chen et al., 2006; Chen \& Zong, 2009), leading to large-scale coronal disturbances like EIT waves (Chen, 2006, 2009), and even triggering a sympathetic CME (Cheng et al., 2005a). A typical CME exhibits the 3-part morphology: a frontal loop, which is followed by a dark cavity with an embedded bright core (e.g., Illing \& Hundhausen, 1985; Dere et al., 1997). It expels approximately $10^{14}-10^{16}$ gram (e.g., Webb et al., 1995) of plasma into interplanetary space with velocity ranging from tens to $3000 \mathrm{~km} \mathrm{~s}^{-1}$ (e.g., St. Cyr et al., 1992; Hundhausen et al., 1994), and with kinetic energy up to $\sim 10^{31}$ erg (Burkepile et al., 2004). At the same time, magnetic field and its intrinsic magnetic helicity are ejected into interplanetary space, which plays an essential part in completing the global magnetic field 
reversal between successive solar cycles (e.g., Zhang \& Low, 2005). They can potentially give rise to hazardous terrestrial effects, such as solar energetic particles (Reames et al., 1999), type II radio burst (Gopalswamy et al., 2009), geomagnetic storms (e.g., Gosling et al., 1990), ionosphere disturbance, and polar aurorae. The information of the magnetic field, mass, and velocity of CMEs is very important since they determine the geomagnetic effectiveness (Gopalswamy et al., 2007).

As a special type, those surrounding the occulting disk, i.e., with an apparent angular width of $360^{\circ}$, are called full halo CMEs (Howard et al., 1982). Compared with normal events with apparent widths between $20^{\circ}$ and $120^{\circ}$, they are generally believed to be nothing special except that they propagate in a direction close to the Sun-Earth line, either toward or away from the Earth. However, it has been noticed that the average apparent velocity of halo CMEs is fairly higher than that of normal CMEs (Webb et al., 1999). For example, Yashiro et al. (2004) compared 7000 events in the period from 1996 to 2002, and found that the average apparent velocity of halo CMEs is twice larger than that of normal CMEs. Such a difference made Lara et al. (2006) propose that halo CMEs are of a special type. Realizing that whitelight emission of CMEs comes mainly from the Thomson-scattering of photospheric radiation, which is much weaker for plasma near the Sun-Earth line than that near the plane of the sky at the same projected heliocentric distance, Andrews (2002) suggested that there exists a mass cut-off, above which CMEs are bright enough to be detected, while many dim, presumably slow events, are missed by coronagraphs. This would make the average apparent velocity of halo CMEs much higher than that of the normal type.

Such a conjecture can be validated if slower CMEs are systematically fainter in brightness. For this purpose, Cheng et al. (2005b) studied the relationship between the apparent velocity and the white-light intensity for halo CMEs. The two parameters did show a marked positive correlation, which provides an indirect support for the mass cut-off conjecture. Compared to limb events, halo CMEs should travel a longer distance to be observed in the FOV of coronagraphs, which has two effects making halo CMEs significantly fainter in white light. First, the intensity of the incident emission from the photosphere is lower. Second, the number density of the CME front becomes smaller. Despite that the cross-section of the Thomson scattering of halo CMEs increases (see Billings, 1966), their white-light emission is reduced greatly compared to the limb CMEs events that are observed at the same projected distance in the plane-of-the-sky. Therefore, it is expected that many halo CMEs, especially the slower events, could be so faint that are missed by coronagraphs. In this paper, we collect a sample of 31 limb CMEs free from projection effect during the SOHO Mission to confirm the velocity-brightness relation. A Monte Carlo simulation is further performed to quantitatively testify whether the observed high value of the average velocity of halo CMEs is due to that many slow events are missed by coronagraphs.

This paper is organized as follows. Data analysis and the results are presented in Section 2 . The Monte Carlo simulation and its result are shown in Section 3 , followed by the discussion on the projection effects in Section 4. We summarize the results in Section 5, along with some discussions.

\section{DATA ANALYSIS AND RESULTS}

Located at the inner Lagrangian point $\left(L_{1}\right)$, SOHO has been monitoring the vigorous Sun for 14 years. Three coronagraphs, C1, C2, and C3, which constitute the LASCO instrument, have FOVs of 1.1-3R $\odot$, $2-6 R_{\odot}$, and $4-32 R_{\odot}$, respectively, where $R_{\odot}$ is the solar radius. The routine observations provide a huge database for $\mathrm{CME}$ research.

In order to select limb events, we carefully checked the movies that are composed of LASCO/C2 and EIT (Delaboudinière et al., 1995) difference images from the NASA CME catalog 11 between 1997 January and 2005 December. The events with associated flares or filament eruptions occurring beyond the longitude of $50^{\circ}$ are collected. Moreover, only the CMEs that have definitely loop-shaped leading edge are considered. It should be kept in mind that the bright loop is not a simple tube but the projection of the dense front of three-dimensional bubble-like structure. To select events with clear loop tops, those CME events with their legs being much brighter than the tops are ignored. Besides, some events whose white-light intensity increases with height or can poorly be fitted with a power-law function with height

\footnotetext{
1 http://cdaw.gsfc.nasa.gov/CME_list
} 
Table 1 List of basic properties of the 31 loop-shaped limb CMEs, including the date, time of first appearance in $\mathrm{C} 2 \mathrm{FOV}$, central position angle (CPA), angular width (AW), and the linear velocity $(V)$.

\begin{tabular}{ccccc}
\hline Date & $\begin{array}{c}\text { Time } \\
(\mathrm{UT})\end{array}$ & $\begin{array}{c}\text { CPA } \\
(\text { Deg })\end{array}$ & $\begin{array}{c}\text { AW } \\
(\text { Deg })\end{array}$ & $\begin{array}{c}V \\
\left(\mathrm{~km} \mathrm{~s}^{-1}\right)\end{array}$ \\
\hline $1997 / 09 / 29$ & $18: 30$ & 78 & 99 & 369 \\
$1997 / 12 / 05$ & $08: 27$ & 275 & 98 & 414 \\
$1998 / 01 / 28$ & $14: 56$ & 268 & 74 & 246 \\
$1998 / 02 / 25$ & $23: 27$ & 74 & 65 & 289 \\
$1998 / 11 / 09$ & $01: 54$ & 16 & 94 & 144 \\
$1999 / 05 / 17$ & $00: 50$ & 293 & 113 & 503 \\
$2000 / 08 / 12$ & $15: 54$ & 254 & 117 & 499 \\
$2000 / 08 / 22$ & $23: 06$ & 179 & 59 & 431 \\
$2000 / 11 / 27$ & $23: 54$ & 123 & 57 & 474 \\
$2001 / 01 / 26$ & $16: 06$ & 55 & 111 & 698 \\
$2001 / 05 / 28$ & $23: 50$ & 96 & 41 & 542 \\
$2001 / 06 / 13$ & $00: 06$ & 279 & 62 & 447 \\
$2001 / 08 / 30$ & $09: 50$ & 129 & 86 & 462 \\
$2001 / 09 / 05$ & $16: 06$ & 232 & 107 & 538 \\
$2002 / 01 / 10$ & $00: 30$ & 236 & 61 & 377 \\
$2002 / 03 / 12$ & $23: 54$ & 112 & 82 & 535 \\
$2002 / 03 / 15$ & $02: 06$ & 64 & 65 & 272 \\
$2002 / 04 / 18$ & $06: 26$ & 162 & 64 & 552 \\
$2002 / 09 / 18$ & $14: 54$ & 279 & 99 & 512 \\
$2002 / 11 / 04$ & $12: 30$ & 17 & 114 & 509 \\
$2002 / 11 / 08$ & $11: 30$ & 298 & 69 & 424 \\
$2003 / 01 / 03$ & $11: 30$ & 283 & 88 & 521 \\
$2003 / 01 / 20$ & $18: 30$ & 315 & 105 & 733 \\
$2003 / 03 / 21$ & $10: 54$ & 54 & 66 & 481 \\
$2003 / 12 / 08$ & $13: 31$ & 228 & 68 & 464 \\
$2004 / 05 / 03$ & $00: 50$ & 113 & 112 & 464 \\
$2004 / 07 / 10$ & $13: 54$ & 270 & 78 & 477 \\
$2004 / 08 / 18$ & $17: 54$ & 258 & 120 & 602 \\
$2004 / 08 / 27$ & $09: 30$ & 261 & 70 & 554 \\
$2005 / 03 / 14$ & $08: 00$ & 259 & 105 & 849 \\
$2005 / 09 / 04$ & $14: 48$ & 286 & 86 & 1179 \\
\hline
\end{tabular}

are also excluded (possibly due to that they are undergoing acceleration). As a result, the sample finally consists of 31 well-defined events, whose image quality indices are $\geq 4$ (labeled as "good" events in the CME catalog). To our understanding, the events with high quality appear less diffuse and have sharp contrast to the background corona so that the height-time measurement is more precise. The basic properties, including the observation date, time of first appearance in the $\mathrm{C} 2 \mathrm{FOV}$, central position angle (CPA), angular width (AW), and the linear velocity $(V)$, are listed in Table 1.

Pre-processing of the LASCO/C2 data, such as correction of flat field and removal of dark current, is conducted by using the standard program c2_calibrate.pro in the Solar SoftWare (SSW). For each event, we take the white-light image just before the first appearance in the $\mathrm{C} 2 \mathrm{FOV}$ as base image to get base difference intensity of the CME frontal loop in the ensuing three snapshots.

It is noted that the observed white-light intensity is the emission integrated along the line-of-sight (LOS), which represents the total mass of unit area in the plane-of-the-sky, i.e., column density. We call it integral intensity. In order to better characterize volume density enhancement of the CME leading loop, we define another parameter - normalized intensity, which is the integral intensity divided by the LOS depth in unit of $R_{\odot}$. Since the 3D topology of CMEs is unclear from a single viewing direction, several versions of the cone model have been proposed based on the fact that the apparent angular widths of many CMEs remain almost constant during their propagation in the corona (e.g., Anzer \& Poland, 1979; Fisher \& Munro, 1984; Michałek et al., 2003; Xie et al., 2004). According to Schwenn et al. (2005), the projected geometry that can well reproduce the kinematic properties of CMEs with the cone angle 


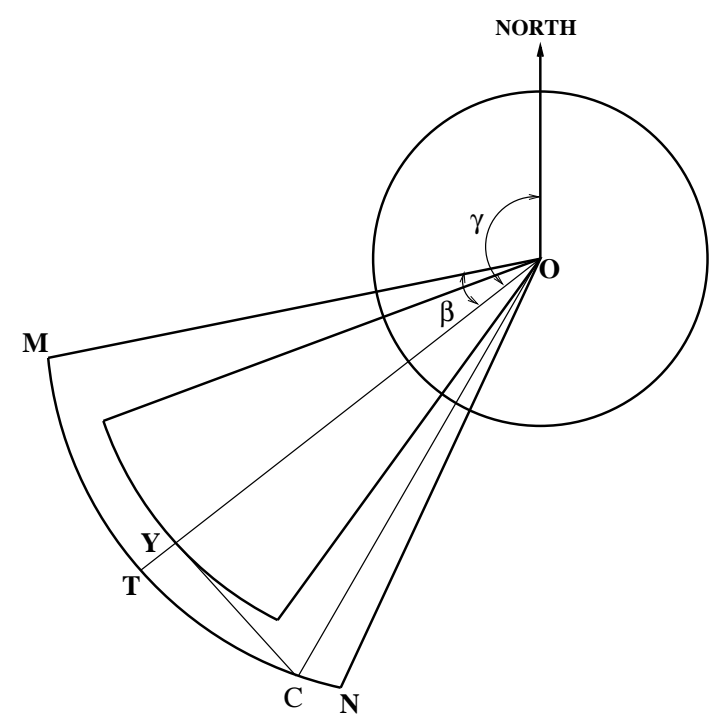

Fig. 1 A sketch of the plane-of-the-sky-projected axisymmetric cone model applied to the analysis of loop-shaped limb CMEs at their early stage of propagation in the corona. The cone OMTNO outlines the CME leading edge, while $\gamma$ and $\beta$ are the central position angle and the angular half-width, respectively. Line $C Y$ perpendicular to $O T$ equals to half LOS depth of $Y$.

between $40^{\circ}$ and $80^{\circ}$ is displayed in Fig. 1 The frontal loop is concentric with the solar disk. The position angle of cone axis $O T$ is denoted by $\gamma$, and the angular half-width by $\beta$, respectively. The LOS depth of white-light emission, which is an unknown parameter, is supposed to be of the same size as the transverse expansion according to the cone model. In the right triangle $\triangle O Y C(Y$ denotes a point along the line $O T$ within the frontal loop), $O C=O T$ and $Y C=\sqrt{(O C)^{2}-(O Y)^{2}}=\sqrt{(O T)^{2}-(O Y)^{2}}$. Under the axisymmetry assumption of the CME 3D morphology, the LOS depth at $Y$ equals to $2 Y C$.

For each CME, the normalized intensity of the loop-top at three moments is obtained and fitted with a power-law function $I_{n} \sim r^{-k}$, where $r$ and $k$ are heliocentric distance and the power index, respectively. As an example, we plot the variation of the normalized intensity $I_{n}$ with $r$ for the 2000 August $22 \mathrm{CME}$ event in Fig. 22, which corresponds to $k=3.60$. In the same way, we derive $k$ for each loop-like event based on near-perfect power-law regression. The distribution of the index, which is averaged at 3.85, is shown in Fig. 3 i.e., on avarage, $I_{n}$ decreases with the heliocentric distance $r$ as $I_{n} \sim r^{-3.85}$. As mentioned in Section 1, two factors contribute to the decrease of $I_{n}$ with $r$. One is the incident emission from the photosphere, the other is the number density of the CME front. It is known that the incident emission decreases with $r$ as $r^{-2}$, therefore, it is derived from the 31 limb events that the number density of the CME frontal loop decreases with height as $\sim r^{-1.85}$, a little more slowly than the first factor. Since a flare- or filament-related CME usually undergoes three phases: initiation phase, fast acceleration phase in the inner corona $\left(\leq 3 R_{\odot}\right)$, and propagation phase with constant speed (Zhang et al., 2001, 2004), the white-light intensity interpolated at $3 R_{\odot}$, both before and after normalization, is calculated to check the relation between CME velocity and brightness. For the 2000 August 22 event, the integral and normalized intensity at $3 R_{\odot}$ is $1.70 \times 10^{-9} \mathrm{msb}$ and $4.47 \times 10^{-10}$ $\mathrm{msb}$, respectively. Here, "msb" is short for "mean solar brightness".

The relation between CME velocity and integral intensity at the frontal loop center (which is also the brightest point) is shown in the left panel of Fig. 4 A clear tendency is seen that the integral intensity of the CME leading loop increases with the CME velocity. The correlation coefficient is as high as 0.62 . Apparently, the 31 limb CMEs all fall in the domain bracketed by the two parallel solid lines in the panel. 


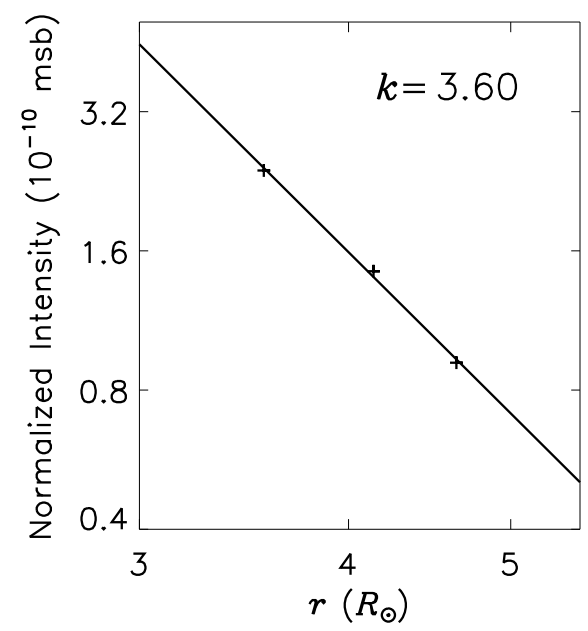

Fig. 2 Variation of the normalized intensity $I_{n}$ of the CME leading edge with the heliocentric distance $r$ for the 2000 August 22 event. The variation is fitted with a power-law profile $I_{n} \sim r^{-k}$, where $k=3.60$.

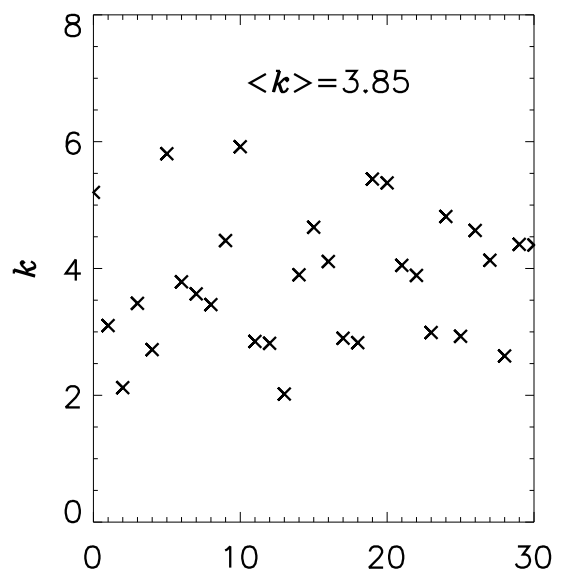

Fig. 3 Scatter plot of the power index $k$ in the 31 loop-like events. The average value of $k$ is 3.85 .

The relation between CME velocity and the normalized intensity at the frontal loop center is shown in the right panel of Fig. 4 . The correlation coefficient increases to 0.72 after normalization. Moreover, the scattering of the data points is reduced compared with the left panel.

The positive correlation between the white-light intensity and the velocity of the limb events confirms the result of Cheng et al. (2005b), and provides indirect evidence in favor of the conjecture of 

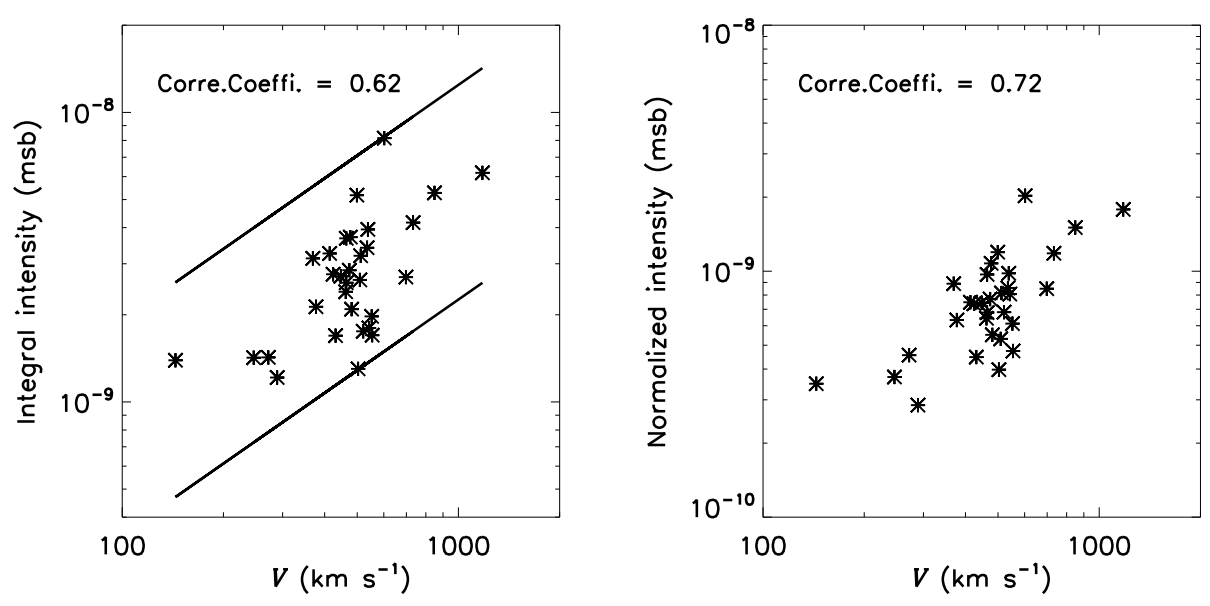

Fig. 4 Scatter diagrams showing the relationship between the velocity and the integral (Left panel) and normalized (Right panel) intensity at the loop center of the 31 sample events. The solid lines bordering the data points in the left panel are parallel to the fitted line from these points. The correlation coefficients are displayed at the upper left of the panels.

Andrews (2002), who proposed that the high average halo CME velocity is due to that some slow events are neglected by coronagraphs. To quantitatively justify this viewpoint, we carry out a Monte Carlo simulation in the next session on the basis of the above correlation.

\section{MONTE CARLO SIMULATION}

As mentioned in Section 1 the white-light emission scattered by the CME front in the halo events would be greatly reduced, compared to limb events. Once the white-light intensity falls below the fluctuation level of the background solar wind, it is likely that a halo CME event is missed by coronagraphs. In this section, using Monte Carlo simulation of 20000 artificial CMEs, we attempt to quantitatively estimate how these effects change the average velocity of the observed halo CMEs.

Figure 5 depicts the geometry of a limb (left panel) and a halo (right panel) CME, based on which we now check how a limb event changes in the apparent velocity and the white-light intensity when it is observed as a halo event. From the geometry, it is easy to see that the apparent velocity decreases by a factor of $\sin \beta$ when a limb CME is observed face-on as a halo event, where $\beta$ is the true angular halfwidth. Line $P Q$ in each panel stands for the longest LOS depth of the cone shell. The integral intensity in the limb and halo cases are labeled with $I_{L}$ and $I_{H}$, respectively.

The change in brightness caused by Thomson-scattering effect is a little complicated. For comparability, the white-light intensity is calculated at a projected distance of $3 R_{\odot}$ for both limb and halo events. Note that the real heliocentric distance of halo CME become $3 R_{\odot} / \sin \beta$. The frontal loop is assumed to have a radial width of $0.8 R_{\odot}$ at $3 R_{\odot}$ and increases by $1 / \sin \beta$. The integral formula for the LOS white-light intensity (Billings, 1966) is then expressed as

$$
I=\frac{1}{2} \pi \sigma_{0} J_{0} R \int_{P}^{Q} n\left[(1-u)\left(2 C \cos ^{-2} \theta-A\right)+u\left(2 D \cos ^{-2} \theta-B\right)\right] d \theta,
$$

where the density $n$ is equal to $n_{3}$ for the limb case and to $n_{3}(1 / \sin \beta)^{-1.85}$ for the halo case, $n_{3}$ is the plasma density of the CME front at $r=3 R_{\odot}, A=\cos \Omega \sin ^{2} \Omega, B=-0.125\left[1-3 \sin ^{3} \Omega-\right.$ $\left.\frac{\cos ^{2} \Omega}{\sin \Omega}\left(1+3 \sin ^{3} \Omega\right) \ln \frac{1+\sin \Omega}{\cos \Omega}\right], C=\left(4-3 \cos \Omega-\cos ^{3} \Omega\right) / 3, D=0.125\left[5+\sin ^{3} \Omega-\frac{\cos ^{2} \Omega}{\sin \Omega}(5-\right.$ 

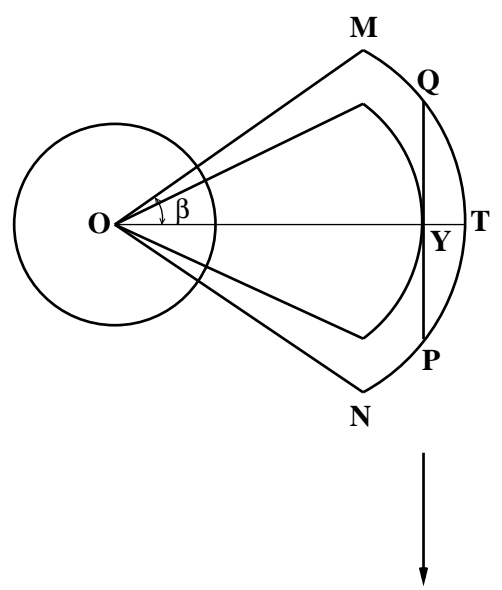

To the observer
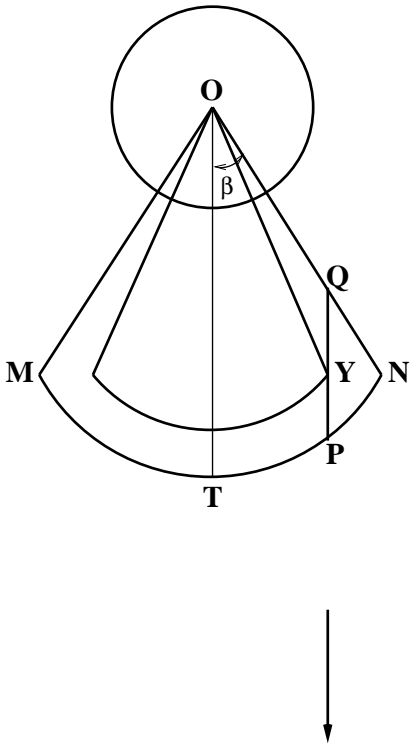

To the observer

Fig. 5 Two sketches illustrating how white-light coronagraphs view CME from an edge-on (Left panel) and face-on (Right panel) perspective.

$\left.\left.\sin ^{3} \Omega\right) \ln \frac{1+\sin \Omega}{\cos \Omega}\right], \sin \Omega=R_{\odot} / r, u=0.6$ is the limb darkening coefficient, $\sigma_{0}$ is the Thomsonscattering cross section, $J_{0}$ the photospheric radiation at the solar surface, and $\theta$ the angular distance between the plane of the sky and the line connecting the Sun center with any point along $P Q$. Besides, we assume that the plasma density is uniform in the frontal loop for simplicity. For different angular half-width $\beta$, the ratio of the integral intensities of the halo and limb CMEs, $I_{H} / I_{L}$, is plotted in Fig. 6. It is seen that for a limb CME, the white-light intensity is reduced by 1-2 orders of magnitude as it is observed as a halo event. From the figure, we can also infer that the wider a halo CME really extends, the higher likelihood it would be detected. Such a result is consistent with Fainshtein (2006), who reported that the real angular widths of halo CMEs with an average value of $>60^{\circ}$ are relatively larger than that of normal CMEs.

In the next step, we perform a Monte Carlo simulation to generate a sample of 20000 artificial limb CMEs that follow the real velocity and angular width distributions. Generally, both the CME velocity and the angular width suffer from the projection effects. In order to eliminate the projection effects, Burkepile et al. (2004) studied the statistical properties of limb CMEs, whose observed velocity and angular width are almost real. Using their data set, we display the velocity distribution of the 72 events with velocity $\geq 100 \mathrm{~km} \mathrm{~s}^{-1}$ in the left panel of Fig. 7. Similar to Aoki et al. (2003), the profile is fitted with a log-normal function, which results in $f_{V}=0.19 \exp \left[-(\ln V-5.76)^{2} / 0.48\right]+0.01$ as displayed by the dashed line. The angular width distribution of the 105 events with $\beta \geq 10^{\circ}$ is illustrated in the right panel of Fig. 7 The histogram is fitted with a Gaussian function $f_{\beta}=0.14 \exp [-(2 \beta-$ $\left.41.95)^{2} / 7.02\right]+0.03$, as shown by the dashed line. The 20000 artificial CMEs are assumed to follow these distributions, with the average velocity being $523 \mathrm{~km} \mathrm{~s}^{-1}$ and the average angular width being $53.8^{\circ}$, respectively.

Then, for each velocity interval, data points with different angular widths are randomly distributed within the two bounding lines shown in the left panel of Fig. 4. The 20000 artificial limb CMEs are scatter-plotted as red crosses in Fig. 8 in the case of $k=3.85$. Note that only the randomly selected $10 \%$ of the data points are shown in order to make the diagram clear. The thick solid horizontal line marks the $3 \sigma$ level of the white-light noise of the background solar corona at $3 R_{\odot}$, below which a 


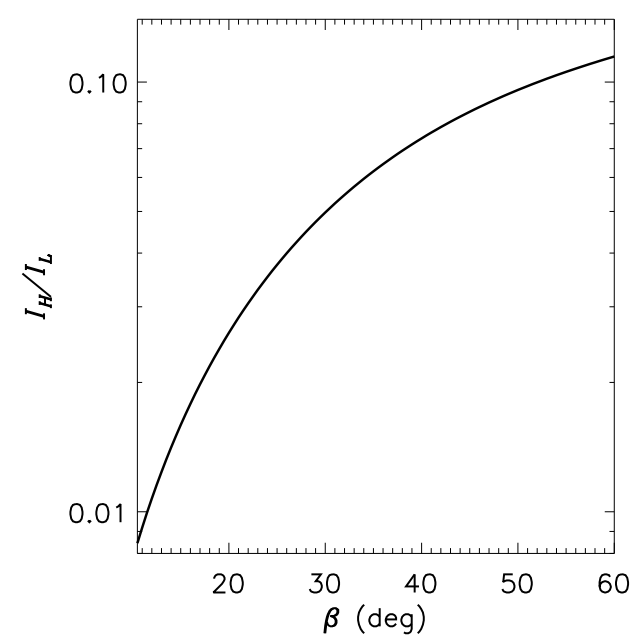

Fig. 6 The ratio of integral white-light intensity of halo CME to that of normal CME as a function of $\beta$ at a projected heliocentric distance $3 R_{\odot}$.
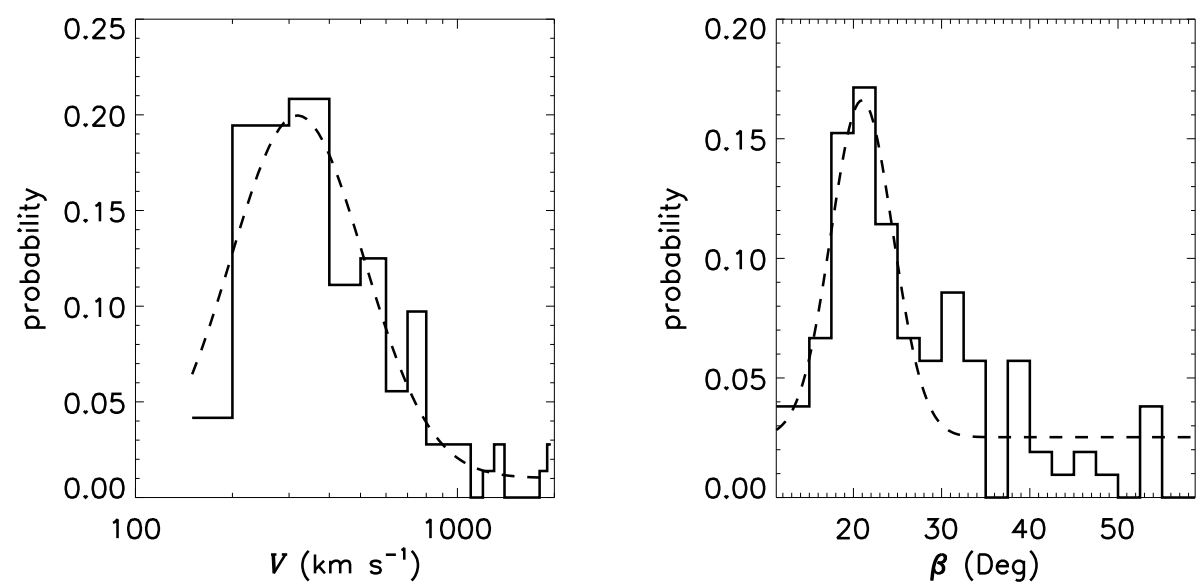

Fig. 7 Velocity (Left panel) and angular half-width (Right panel) distributions of limb CMEs. The histograms are from the observational results of Burkepile et al. (2004), while the dashed lines are out fitted profiles, a log-normal function for the velocity and a Gaussian function for the angular half-width.

data point is considered as unobservable. Here $\sigma=2.33 \times 10^{-10} \mathrm{msb}$ is the standard deviation of the background fluctuations in the LASCO/C2 images at $3 R_{\odot}$. For each data point, as the event is observed as a halo CME, its apparent (plane-of-the-sky) velocity decreases by a factor of $\sin \beta$, while its white-light intensity drops by $I_{H} / I_{L}$ (see Fig. 6). After such corrections, the new data points of the corresponding halo CMEs are plotted as blue diamonds. It is found that if the artificial events are 


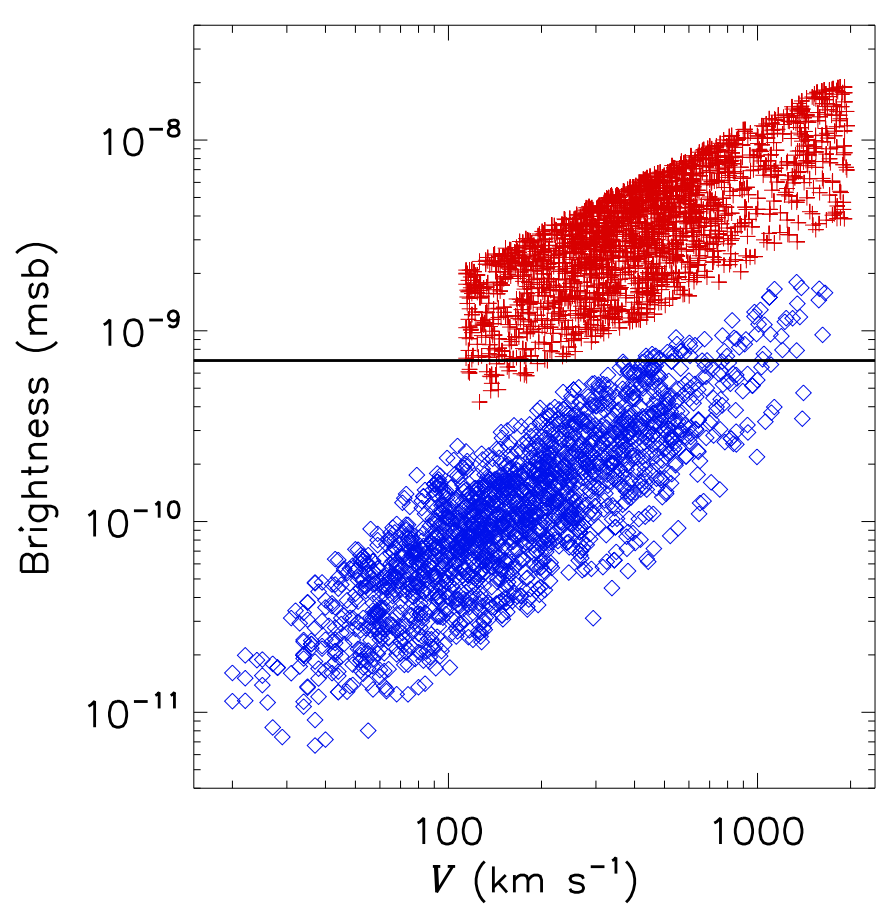

Fig. 8 Scatter diagrams of the randomly selected one tenth of the 20000 artificial events. Red crosses and blue diamonds represent limb and halo events. The horizontal line marks the $3 \sigma$ level of the background solar wind fluctuations at $3 R_{\odot}$.

Table 2 Parameter survey of the average velocity of halo CMEs $\left(\bar{V}\right.$, in unit of $\left.\mathrm{km} \mathrm{s}^{-1}\right)$.

\begin{tabular}{c|ccc}
\hline & $h=0.5 R_{\odot}$ & $h=0.8 R_{\odot}$ & $h=1.0 R_{\odot}$ \\
\hline$k=3.00$ & 894 & 673 & 600 \\
$k=3.85$ & 1144 & 922 & 826 \\
$k=4.00$ & 1161 & 954 & 861 \\
\hline
\end{tabular}

observed as halo CMEs, the white-light intensity of a majority of the sample falls below the $3 \sigma$ level, and therefore the corresponding CMEs are considered to be "missed" by the LASCO coronagraph. The average velocity $(\bar{V})$ of the "visible" halo CMEs is calculated to be $922 \mathrm{~km} \mathrm{~s}^{-1}$.

There are several assumptions about the properties of the CME frontal loop in the simulation, e.g., the radial width $(h)$, the density variation with height $\left(r^{-(k-2)}\right)$, and so on, where the density variation with height was derived from the 31 well-defined CMEs. To show how the result is affected by the assumptions, we performed a series of simulations with different $h$ and $k$. The corresponding $\bar{V}$ is displayed in Table 2] with the unit of $\mathrm{km} \mathrm{s}^{-1}$.

\section{PROJECTION EFFECT}

As one of the puzzles in CME research, it is recognized that halo CMEs are much faster than normal events, although in principle, the difference between halo and limb events is only the direction of prop- 

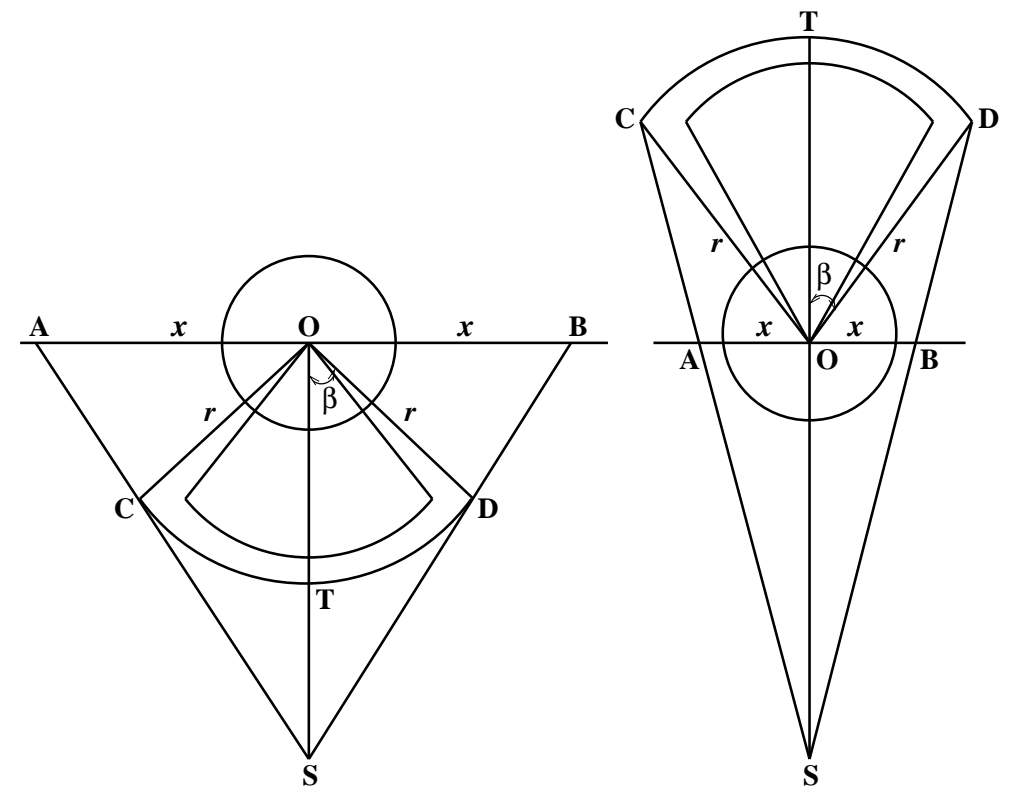

Fig. 9 The observing geometry of halo CMEs in the front-side (left panel) and back-side (right panel) cases. $A B$ signifies the plane of the sky, and $S$ the observing spacecraft (SOHO).
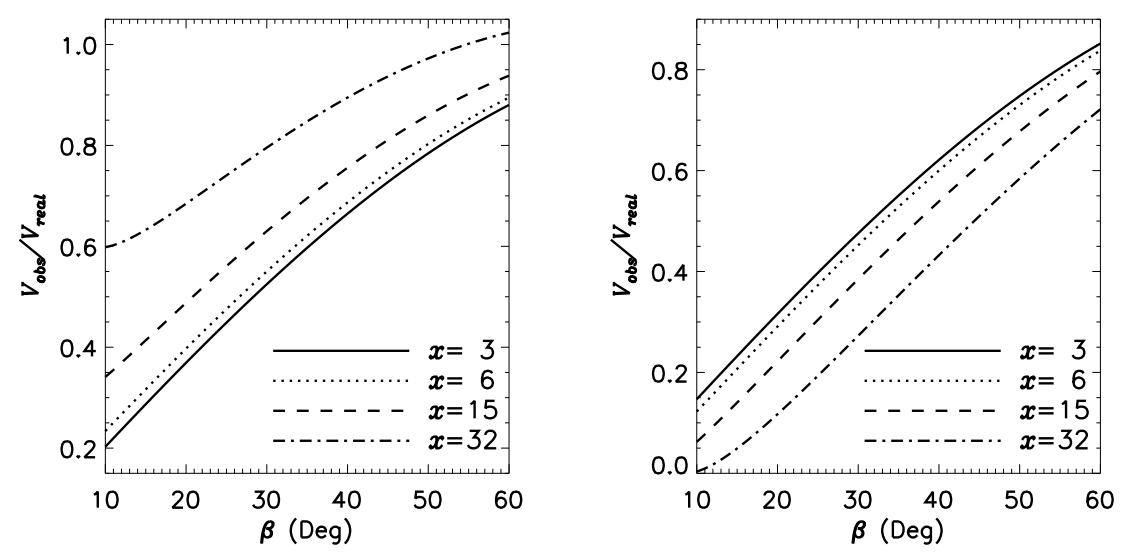

Fig. 10 The relationship between $V_{\text {obs }} / V_{\text {real }}$ and $\beta$ for fore-side (Left panel) and back-side (Right panel) halo CMEs. Different line styles (solid, dotted, dashed, and dash-dotted) represent the relationship at different projected heliocentric distances $\left(3,6,15\right.$, and $\left.32 R_{\odot}\right)$.

agation. Here we discuss whether the simple projection effect is related to the high velocity puzzle of halo CMEs.

Since halo CMEs propagate either toward or away from the Earth as illustrated in Fig. 9, the leading edge (e.g., point $D$ ) is projected to the plane of the sky (i.e., point $B$ ) to calculate the propagation velocity of the CME. The left/right panel of Fig. 9 depicts the propagation geometry for a front-side/back- 
side CME with real angular half-width $\beta$. The real heliocentric distance of the leading loop, $r$, is equal to the length of $O D$, while the apparent heliocentric distance, $x$, is equal to the length of $O B$. The SOHO spacecraft, which is located at $L_{1}$ with a heliocentric distance of $L=212 R_{\odot}$, is marked with $\mathrm{S}$. According to the geometrical relation, the ratio between the apparent velocity $\left(V_{\mathrm{obs}}\right)$ and the real velocity $\left(V_{\text {real }}\right)$ as a function of $\beta$ and $x$ is expressed as

$$
V_{\text {obs }} / V_{\text {real }}=\frac{d x}{d t} / \frac{d r}{d t}= \begin{cases}{[1+x /(L \tan \beta)]^{2} \sin \beta,} & \text { front-side, } \\ {[1-x /(L \tan \beta)]^{2} \sin \beta,} & \text { back-side. }\end{cases}
$$

The relationship between the velocity ratio and $\beta$ in both cases is displayed in Fig. 10 (left panel for the front-side events and right panel for the back-side), where different lines correspond to the relation at different apparent heliocentric distances within LASCO FOV. It is seen that for both types of events, the apparent velocity is always smaller than the real one within the LASCO FOV. For the front-side halo CMEs, the velocity-reducing factor increases from 0.2 to 0.88 as $\beta$ increases from $10^{\circ}$ to $60^{\circ}$ when the projected heliocentric distance is $3 R_{\odot}$. At a larger distance, the reducing factor increases. For the back-side events, the reducing factor is slightly smaller than that in the front-side case at $3 R_{\odot}$. However, the factor decreases with distance as indicated in the right panel.

The above theoretical analysis reveals that the projection effect results in a smaller apparent velocity of halo CMEs. Therefore, the projection effect cannot resolve the high velocity puzzle of halo CMEs. Such a result is consistent with Michałek et al. (2003) and Howard et al. (2008), who found that the CME velocity becomes higher after correcting the projection effect. Combined radio, in situ, and whitelight observations of CME/shocks have also revealed that the plane-of-the-sky speeds for fast halo CMEs are always less than or equal to the initial radial speeds (Reiner et al., 2007).

\section{SUMMARY}

In this paper we first analyzed the relation between the white-light intensity and the propagation velocity of $31 \mathrm{limb}$ CMEs observed clearly by the SOHO/LASCO coronagraph. It is confirmed that slower CMEs tend to be weaker in the white-light brightness, meaning that they carry less plasma. We also studied the normalized intensity evolution of the CME leading edge along with the radial distance $r$ and fitted it with a power-law function, $I_{n} \sim r^{-k}$. It is found that the power index $k$ is averaged around 3.85. Considering that the incident emission from the photosphere decreases as $r^{-2}$, it means that the plasma density of a CME frontal loop, $n$, decreases with $r$ roughly as $r^{-1.85}$. Although the cross-section of Thomson scattering increases as a limb CME is observed as a halo CME, the abrupt decreases of the incident light from the photosphere and $n$ with $r$ lead to that the white-light intensity of halo CMEs would be very weak since they should travel a longer distance to be observed by coronagraphs.

As a further quantitative investigation to resolve the high velocity puzzle for halo CMEs, we performed a Monte Carlo simulation of 20000 artificial CMEs with velocity and angular width distributions derived from limb CMEs. The Thomson-scattering intensity is calculated for both limb and halo events. The simulation indicates that if the limb CMEs with angular width between $20^{\circ}$ and $120^{\circ}$ are observed as full halo events propagating along the Sun-Earth line, a majority of the slower events become so weak that their intensity is comparable to the fluctuation of the background solar wind, implying that they would fail to be identified by coronagraphs. The average velocity of the "detectable" halo CMEs is $\sim 922 \mathrm{~km} \mathrm{~s}^{-1}$, which agrees perfectly with previous statistical result (Michałek et al., 2003). We believe that the missing of many slow halo CMEs can well explain the high velocity puzzle of halo CMEs. As a proof of statement that some CMEs may be neglected even by the state-of-art coronagraphs, here we mention the 2007 December 7 event, which was observed by STEREO (Solar TErrestrial RElations Observatory) A satellite (Kaiser, 2005), but completely missed by STEREO B since the event was more face-on to STEREO B (Ma et al., 2009). Statistical work using multi-directional data is of help to clarify how many faint halo CMEs are missed and testify our conclusion.

It should also be emphasized that the halo CMEs in the simulation are full halo ones that originate at the disk center and propagate along the Sun-earth line. For the full halo CMEs that propagate aside from the Sun-earth line and partial halo CMEs, the white-light intensity decrease would not be so significant 
as in Fig. 6 Thus, less portion of such events would be missed. In order to quantitatively compare the Monte Carlo simulation with observations, these cases would also be considered in a future work.

Acknowledgements The authors thank J. Zhang and Y. H. Tang for instructive discussions and suggestion throughout the work. We are also grateful to the anonymous referee for suggestions. This research is supported by the Chinese foundations GYHY200706013, 2006CB806302, NSFC (10403003, 10933003, and 10673004). SOHO is a project of international cooperation between ESA and NASA.

\section{References}

Andrews, M. D. 2002, Sol. Phys., 208, 317

Anzer, U., \& Poland, A. I. 1979, Sol. Phys., 61, 95

Aoki, S., Yashiro, S., and Shibata, K. 2003, International Cosmic Ray Conference, 5, 2729

Billings, D. E., A Guide to the Solar Corona, New York: Academic Press, 1966

Burkepile, J. T., Hundhausen, A. J., Stanger, A. L., St. Cyr, O. C., \& Seiden, J. A. 2004, J. Geophys. Res.(space phys.), 109, 3103

Brueckner, G. E., et al. 1995, Sol. Phys., 162, 357

Chen, A. Q., Chen, P. F., \& Fang, C. 2006, A\&A, 456, 1153

Chen, A.-Q., \& Zong, W.-G. 2009, Research in Astronomy and Astrophysics, 9, 470

Chen, P. F. 2006, ApJ, 641, L153

Chen, P. F. 2009, ApJ, 698, L112

Cheng, J.-X., Fang, C., Chen, P.-F., \& Ding, M.-D. 2005, Chinese Journal of Astronomy and Astrophysics, 5, 265

Cheng, J. X., Yeh, C. T., Ding, D. M., \& Chen, P. F. 2005, Proc. of IAU Symp. 226, p. 112

Dere, K. P., et al. 1997, Sol. Phys., 175, 601

Delaboudinière, J.-P., et al. 1995, Sol. Phys., 162, 291

Domingo, V., Fleck, B., \& Poland, A. I. 1995, Sol. Phys., 162, 1

Fainshtein, V. G. 2006, Geomagnetism and eronomy/Geomagnetizm i Aeronomiia, 46, 339

Fisher, R. R., \& Munro, R. H. 1984, ApJ, 280, 428

Gopalswamy, N., Yashiro, S., \& Akiyama, S. 2007, Journal of Geophysical Research (Space Physics), 112, 6112

Gopalswamy, N., et al. 2009, Sol. Phys., 80

Gosling, J. T., Bame, S. J., McComas, D. J., \& Phillips, J. L. 1990, Geophys. Res. Lett., 17, 901

Howard, R. A., Michels, D. J., Sheeley, N. R., Jr., \& Koomen, M. J. 1982, ApJ, 263, L101

Howard, T. A., Nandy, D., \& Koepke, A. C. 2008, Journal of Geophysical Research (Space Physics), 113, 1104

Hundhausen, A. J., Burkepile, J. T., \& St. Cyr, O. C. 1994, J. Geophys. Res.(space phys.), 99, 6543

Illing, R. M. E., \& Hundhausen, A. J. 1985, J. Geophys. Res.(space phys.), 90, 275

Kaiser, M. L. 2005, Advances in Space Research, 36, 1483

Lara, A., Gopalswamy, N., Xie, H., Mendoza-Torres, E., Pérez-Eríquez, R., \& Michalek, G. 2006,

J. Geophys. Res.(space phys.), 111, 6107

Ma, S. L. et al. 2009, ApJ, submitted

Michałek, G., Gopalswamy, N., \& Yashiro, S. 2003, ApJ, 584, 472

Reames, D. V., Ng, C. K., \& Tylka, A. J. 1999, Geophys. Res. Lett., 26, 3585

Reiner, M. J., Kaiser, M. L., \& Bougeret, J.-L. 2007, ApJ, 663, 1369

Schwenn, R., dal Lago, A., Huttunen, E., \& Gonzalez, W. D. 2005, Annales Geophysicae, 23, 1033

St. Cyr, O. C., Hundhausen, A. J., \& Burkepile, J. T. 1992, Proc. of the First SOHO Workshop: Coronal Streamers,

Coronal Loops, and Coronal and Solar Wind Composition, p. 125

Tousey, R. 1973, Space Research XIII, Vol. 2, p. 713

Webb, D. F., Howard, R. A., \& Jackson, B. V. 1995, Solar Wind VIII, 97

Webb, D. F., St. Cyr, O. C., Plunkett, S. P., Howard, R. A., \& Thompson, B. J. 1999, Bulletin of the American Astronomical Society, 31, 853

Xie, H., Ofman, L., \& Lawrence, G. 2004, J. Geophys. Res.(space phys.), 109, 3109

Yashiro, S., Gopalswamy, N., Michalek, G., St. Cyr, O. C., Plunkett,S. P.,Rich, N. B., \& Howard, R. A. 2004, J. Geophys. Res.(space phys.), 109, 7105 
Zhang, J., Dere, K. P., Howard, R. A., Kundu, M. R., \& White, S. M. 2001, ApJ, 559, 452

Zhang, J., Dere, K. P., Howard, R. A., \& Vourlidas, A. 2004, ApJ, 604, 420

Zhang, M., \& Low, B. C. 2005, ARA\&A, 43, 103 